\title{
New small-size peptides modulators of the exosite of BACE1 obtained from a structure-based design
}

Lucas J. Gutierrez ${ }^{\mathrm{a}}$, Emilio Angelina ${ }^{\mathrm{a}, \mathrm{b}}$, Andrea Gyebrovszkic ${ }^{\mathrm{c}}$, Lívia Fülöp ${ }^{\mathrm{c}}$, Nelida Peruchena ${ }^{\mathrm{a}}$, Héctor A. Baldoni ${ }^{\mathrm{d}, \mathrm{e}}$,

${ }^{a}$ Laboratorio de Estructura Molecular y Propiedades, Área de Química Física, Departamento de Químicam, Facultad de Ciencias Exactas y Naturales y Agrimensura, Universidad Nacional del Nordeste, Av. Libertad 5470, Corrientes 3400, Argentina; ${ }^{b}$ Instituto Multidisciplinario de Investigaciones Biológicas de San Luis (IMIBIO-SL, CONICET), Ejercito de Los Andes 950, 5700 San Luis, Argentina; ${ }^{c}$ Department of Medical Chemistry, University of Szeged, H-6720 Szeged, Dóm tér 8., Hungary; ${ }^{d}$ Facultad de Quimica,

10 Bioquímica y Farmacia, Universidad Nacional de San Luis, Chacabuco 917, 5700 San Luis, Argentina; 'Instituto de Matemática Aplicada San Luis (IMASL,CONICET), Italia 1556, 5700 San Luis, Argentina

Communicated by Ramaswamy H. Sarma

(Received 13 October 2015; accepted 19 January 2016)

We report here two new small-size peptides acting as modulators of the $\beta$-site APP cleaving enzyme 1 (BACE1) exosite. Ac-YPYFDPL-NH2 and Ac-YPYDIPL-NH2 displayed a moderate but significant inhibitory effect on BACE1. These peptides were obtained from a molecular modeling study. By combining MD simulations with ab initio and DFT calculations, a simple and generally applicable procedure to evaluate the binding energies of small-size peptides interacting with the exosite of the BACE1 is reported here. The structural aspects obtained for the different complexes were analyzed providing a clear picture about the binding interactions of these peptides. These interactions have been investigated within the framework of the density functional theory and the quantum theory of atoms in molecules using a reduced model. Although the approach used here was traditionally applied to the study of noncovalent interactions in small molecules complexes in gas phase, we show, through in this work, that this methodology is also a very powerful tool for the study of biomolecular complexes, providing a very detailed description of the binding event of peptides modulators at the exosite of BACE1.

Keywords: Alzheimer's disease; $\beta$-secretase; exosite-modulators; molecular modeling; molecular simulations

\section{Introduction}

Alzheimer's disease (AD) is the most common form of dementia affecting $6-10 \%$ of people over the age of 65 (Clippingdale, Wade, \& Barrow, 2001). Several possible molecular mechanisms may initiate AD. However, considerable genetic and biochemical evidence suggests that the amyloid $\beta$-peptide $(A \beta)$ misfolding oligomerization and accumulation in the brain is the primary cause of the neuronal dysfunction (Hardy \& Selkoe, 2002).

The most widely accepted theory regarding the etiology of AD is known as the "amyloid hypothesis" which features the $A \beta$ as the central pathological agent. This hypothesis posits that pathology initiates because of an imbalance in $\mathrm{A} \beta$ production and/or clearance, which may result from altered expression or processing of amyloid precursor protein (APP) or changes in $\mathrm{A} \beta$ metabolism (Hardy \& Selkoe, 2002). Development of therapies that reduce amyloidogenic processing holds great promise but has not yet proven successful clinically (Cummings, 2010). The aspartyl protease $\beta$-site APP cleaving enzyme
1 (BACE1) is the primary $\beta$-secretase in the brain, making it a prime candidate for $\mathrm{AD}$ therapeutics. Many potent BACE1 inhibitors (INHs) have been described, but only recently some have been reported that are able to cross the blood-brain barrier in sufficient quantities to produce the biological effect (Varghese, 2010). In fact, most of the aspartyl protease INHs in current clinical use today are peptidomimetics that target the catalytic active site of the enzyme. While these drugs have demonstrated usefulness for treating systemic viral infections, their meager ability to cross the blood-brain barrier may prove to be an obstacle to the use of such compounds for treating diseases of the central nervous system, such as AD.

In addition to the active site, BACE1 contains an additional binding pocket, termed exosite, which engages substrates in the vicinity of the active site (Gutierrez, Enriz, \& Baldoni, 2010; Kornacker et al., 2005). Previously, we reported that this binding pocket can contribute significantly to the stabilization of the enzyme-substrate

\footnotetext{
*Corresponding author. Email: denriz@unsl.edu.ar 
binary complex by providing important structural determinants of interaction (Gutierrez et al., 2010). Additionally, this exosite can act as allosteric regulator of the enzyme activity, causing an augmentation or diminution of the enzyme's catalytic reactivity. Kornacker et al. reported that some small peptides bind to the exosite of BACE1 in a manner that is unaffected by active site ligand occupancy (Kornacker et al., 2005). Peptides that bind in this exosite are able to inhibit the ability of BACE1 to hydrolyze its natural protein substrate, APP. It must be pointed out that the effect of these peptides is at the exosite of the enzyme and not in the catalytic site; therefore, we can expect a modulating effect and not necessarily a potent inhibitory activity. Having clarified this concept, from now we will use indistinctly the terms modulator or inhibitor.

Experimental evidences showed that it is possible to inhibit the catalytic activity of BACE1 by targeting its exosite (Atwal et al., 2011; Zhou et al., 2011). These authors have designed a bispecific $\mathrm{mAb}$; one arm comprising a low-affinity anti-transferrin Fab fragment (antiTfR), and the other arm comprising the high-affinity anti-BACE1 Fab fragment (anti-BACE1). They showed by X-ray crystallography that these antibodies inhibit BACE1 activity by binding the BACE1-exosite.

Our research group reported the exact location of the BACE1-exosite using a blind docking study demonstrating the utility of this technique (Gutierrez et al., 2010). More recently, we have reported the structural and thermodynamic characteristics of this exosite by utilizing the technique of alanine scanning (Gutiérrez, Andujar, Enriz, $\&$ Baldoni, 2013) Once we possess such information, the main objective of the present work is to obtain new peptides modulators of the exosite of BACE1. To achieve this objective, we performed in the first step a molecular modeling study on a series of peptides previously reported by Kornaker et al. with inhibitory properties on the exosite of BACE1. In this study, we combined MD simulations with quantum mechanies (semiempirical and DFT) calculations. In order to better evaluate the molecular interactions (MI) between the peptides and the BACE1-exosite, a quantum theory of atoms in molecules (QTAIM) analysis of the different complexes was also carried out. The principal goal of such study was to perform a comparative analysis among the stabilizing and destabilizing interactions involved in the different complexes in order to obtain a structure-affinity relationship. A reasonable correlation between $\hat{\sum} \rho_{(r)}$ obtained from QTAIM calculations and experimental data (Kornacker here. On the basis of this crucial information in the second step of our study, we have designed, synthesized, and tested new structurally related peptides that possess the desired modulatory activity.

\section{Materials and methods}

\subsection{Experimental section}

2.1.1. Solid-phase peptide synthesis of Ac-YPYFDPL$\mathrm{NH}_{2}$ and $\mathrm{Ac}-\mathrm{YPYDIPL}-\mathrm{NH}_{2}$

The peptides were synthesized on an Fmoc-Rink Amide $\mathrm{AM}$ resin $\left(.3 \mathrm{mmol} \mathrm{g}{ }^{-1}\right)$, on a $.3 \mathrm{mmol}$ scale with a standard Fmoc-chemistry, applying DCC/HOBt as coupling reagents in a mixture of DCM/DMF. The efficiency of the couplings was monitored with ninhydrin test. The peptides were acetylated with a mixture containing $10 \%$ $(\mathrm{v} / \mathrm{v})$ acetic acid anhydride and 5\% (v/v) DIEA in DCM.

The peptides were cleaved from the resin with a mixture of TFA/ $\mathrm{H}_{2} \mathrm{O} / \mathrm{TIS}$ (95:2.5:2.5 volume ratio) at $0{ }^{\circ} \mathrm{C}$ for $.25 \mathrm{~h}$, and at room temperature for $2 \mathrm{~h}$. The TFA was removed in vacuo. The peptides were dissolved in $40 \%$ acetonitrile in water and lyophilized. Crude peptides were purified by RP-HPLC on a Phenomenex Luna C18 column $(250 \times 21.20 \mathrm{~mm}$, pore size: $100 \AA$, particle diameter: $10 \mu \mathrm{m}$, flow rate: $3 \mathrm{ml} / \mathrm{min}$ ). The solvent system consisted of . $1 \%$ TFA in water (A), and .1\% TFA in $80 \%$ acetonitrile (B). Purity of the peptides was checked by analytical RPHPLC equipped with a Phenomenex Luna C18 column $(250 \times 4.6 \mathrm{~mm}$, pore size: $100 \AA$, particle diameter: $5 \mu \mathrm{m}$, flow rate: $1.2 \mathrm{ml} / \mathrm{min}$ ) and by ESI-MS in a positive ion mode. The HPLC and MS results for both peptides are shown in supplementary material (Figures $1 \mathrm{~S}$ and $2 \mathrm{~S}$ ).

\subsubsection{BACE1 fluorescence resonance energy transfer} (FRET) assay

The BACE1 FRET assay kit was purchased from Sigma (Catalog Number CS0010). BACE1 activity assays were carried out following the protocol of manufacturer. The BACE1 assay was carried out on Perkin Elmer luminescence spectrometer LS50B, excitation $320 \mathrm{~nm}$ (slit $12 \mathrm{~nm}$ ) and emission $405 \mathrm{~nm}$ (slit $12 \mathrm{~nm}$ ).

\subsection{Molecular modeling}

Calculations were carried out in three steps. In the first step, we performed preliminary molecular dynamic simulations of BACE1 with the peptides listed in Table 1. In the second step, reduced model systems were optimized using quantum mechanics calculations. Semiempirical (PM6 and PM6-COSMOS) combined with B3LYP (6$31 \mathrm{G}(\mathrm{d})$ ) calculations were employed in such optimizations. Finally, the ten complexes obtained in the previous steps were further analyzed from a QTAIM study.

\subsubsection{System setup}

The starting geometry used in this work was the called as "C4" in our previous work in which we reported the 
Table 1. Sequence for exosite-binding peptides of BACE1.

\begin{tabular}{|c|c|}
\hline INH & Sequence $^{\mathrm{a}}$ \\
\hline 1 & Ac-Pro $_{10}-\mathrm{Leu}_{11}-\mathrm{Pro}_{12}-\mathrm{NH}_{2}$ \\
\hline 2 & Ac-Ile $_{9}-$ Pro $_{10}-\mathrm{Leu}_{11}-\mathrm{Pro}_{12}-\mathrm{NH}_{2}$ \\
\hline 3 & Ac-Tyr $5-\mathrm{Pro}_{6}-\mathrm{Tyr}_{7}-\mathrm{Phe}_{8}-\mathrm{Ile}_{9}-\mathrm{NH}_{2}$ \\
\hline 4 & Ac-Tyr ${ }_{5}-\mathrm{Pro}_{6}-\mathrm{Tyr}_{7}-\mathrm{Phe}_{8}-\mathrm{Ile}_{9}-\mathrm{Pro}_{10}-\mathrm{NH}_{2}$ \\
\hline 5 & Ac-Tyr ${ }_{5}-\mathrm{Pro}_{6}-\mathrm{Tyr}_{7}-\mathrm{Phe}_{8}-\mathrm{Ile}_{9}-\mathrm{Pro}_{10}-\mathrm{Leu}_{11}-\mathrm{Pro}_{12}-\mathrm{NH}_{2}$ \\
\hline 6 & 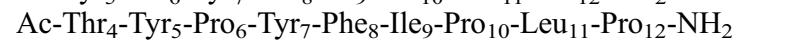 \\
\hline 7 & Ac-Thr ${ }_{3}-\mathrm{Thr}_{4}-\mathrm{Tyr}_{5}-\mathrm{Pro}_{6}-\mathrm{Tyr}_{7}-\mathrm{Phe}_{8}-\mathrm{Ile}_{9}-\mathrm{Pro}_{10}-\mathrm{Leu}_{11}-\mathrm{Pro}_{12}-\mathrm{NH}_{2}$ \\
\hline 8 & Ac-Tyr - Pro $_{6}-\mathrm{Tyr}_{7}-\mathrm{Phe}_{8}-\mathrm{Ile}_{9}-\mathrm{Pro}_{10}-\mathrm{Leu}_{11}-\mathrm{NH}_{2}$ \\
\hline 9 & Ac-Tyr ${ }_{5}-\mathrm{Pro}_{6}-\mathrm{Tyr}_{7}-\mathrm{Phe}_{8}-\mathrm{Asp}_{9}-\mathrm{Pro}_{10}-\mathrm{Leu}_{11}-\mathrm{NH}_{2}$ \\
\hline 10 & Ac-Tyr $5-\mathrm{Pro}_{6}-\mathrm{Tyr}_{7}-\mathrm{Asp}_{8}-\mathrm{Ile}_{9}-\mathrm{Pro}_{10}-\mathrm{Leu}_{11}-\mathrm{NH}_{2}$ \\
\hline
\end{tabular}

${ }^{\mathrm{a}}$ Residue numbering was taken from reference Kornacker et al. (2005).

structural characteristics of the exosite of BACE1 (Gutierrez et al., 2010). In this study, we used the same inhibitor (compound 8) as template; thus the different ligands shown in Table 1 were constructed by adding or removing amino acids from such starting structure.

\subsubsection{Molecular dynamics simulations}

To relax the binding complexes, thirty-nanosecond MD simulations were performed for the different BACE1inhibitor (BACE1-INH) systems using Amber software (Case et al., 2012). The protein force field was taken from ff99SBildn (Lindorff-Larsen et al., 2010).

Each complex BACE1-INH was soaked in a truncated octahedral periodic box of TIP3P water molecules

15 (Jorgensen, Chandrasekhar, Madura, Impey, \& Klein, 1983). The distance between the edges of the water box and the closest atom of the solutes was at least $10 \AA$. $\mathrm{Na}+$ ions were added to neutralize the systems charge. The entire system was subject to energy minimization 20 in two stages to remove bad contacts between the complex and the solvents molecules. Firstly, the water molecules were minimized by holding the solute fixed with harmonic constraints of $100 \mathrm{kcal} / \mathrm{mol} \AA^{2}$ strength. Secondly, conjugate gradient energy minimizations were 25 performed repeatedly four times using positional restraints to all heavy atoms of the receptor with 15, 10,5 , and $0 \mathrm{kcal} / \mathrm{mol} \AA^{2}$. The system was then heated from 0 to $300 \mathrm{~K}$ in $300 \mathrm{ps}$ and equilibrated at $300 \mathrm{~K}$ for another $200 \mathrm{ps}$. After minimization and heating, three independent simulations with length to $10 \mathrm{~ns}$ were performed at a constant temperature of $300 \mathrm{~K}$ and a constant pressure of $1 \mathrm{~atm}$. During minimization and MD simulations, particle mesh Ewald method (Essmann et al., 1995) was employed to treat the long-range elecHydrogen stretching motions were removed using SHAKE algorithm (Ryckaert, Ciccotti, \& Berendsen, 1977) allowing an integration time step of 2 fs and the nonbonded cutoff distance was $8.0 \AA$. The only differ-

ence between replicates was in the initial velocity assignments at the start of the dynamics.

\subsubsection{Binding energy calculations}

The MM-GBSA protocol was applied to each MD trajectory in order to calculate the relative binding energies of the BACE1-INH complexes. The MM-GBSA method was used in a hierarchical strategy, and the details of this method have been presented elsewhere (Kollman et al., 2000). This protocol was applied to 1000 equidistant snapshots extracted from the last $5.0 \mathrm{~ns}$ of each replicate and was used within the one-trajectory approximation. Briefly, the binding free energy $\left(\Delta G_{\text {bind }}\right)$ resulting from the formation of a RL complex between a ligand (L) and a receptor $(R)$ is calculated as

$$
\begin{gathered}
\Delta G_{\text {bind }}=\Delta E_{\mathrm{MM}}+\Delta G_{\mathrm{sol}}-T \Delta S \\
\Delta E_{\mathrm{MM}}=\Delta E_{\text {internal }}+\Delta E_{\text {electrostatic }}+\Delta E_{\mathrm{vdw}} \\
\Delta G_{\mathrm{sol}}=\Delta G_{\mathrm{GB}}+\Delta G_{\mathrm{SA}}
\end{gathered}
$$

where $\Delta E_{\mathrm{MM}}, \Delta G_{\mathrm{sol}}$, and $-T \Delta S$ are the changes in the gas-phase MM energy, the solvation free energy, and the conformational entropy upon binding, respectively. $\Delta E_{\mathrm{MM}}$ includes $\Delta E_{\text {internal }}$ (bond, angle, and dihedral energies), $\Delta E_{\text {electrostatic }}$ (electrostatic), and $\Delta E_{\mathrm{vdw}}$ (van der Waals) energies. $\Delta G_{\text {solv }}$ is the sum of electrostatic solvation energy (polar contribution), $\Delta G_{\mathrm{GB}}$, and the non-electrostatic solvation component (nonpolar contribution), $\Delta G_{\mathrm{SA}}$. Polar contribution is calculated using the GB model, while the nonpolar energy is estimated by solvent accessible surface area. The conformational entropy change $-T \Delta S$ is usually computed by normal-mode analysis, but in this study the entropy contributions were not calculated due to the computational cost involved in such calculations. 


\subsubsection{Constructing the reduced models for the binding} site

The use of model systems to calculate and simulate MI is necessary since the INHs interacting at the exosite of BACE1 constitute a molecular system, too large for accurate Quantum Mechanic Molecular Orbital calculations, and the number of peptides to be screened is large as well. Moreover, a model system representing the binding pocket of BACE1-exosite may be desirable in order to evaluate the ability of the ligands to interact with this exosite. Using a model, complexities due to the rest of the BACE1 enzyme are avoided. The questions which arise are as follows: how can we select only those amino acids involved in the interactions forming the different BACE1-INH complexes? To acquire a more detailed insight into the mechanisms driving the bindings of INHs to the exosite of BACE1, the structure-affinity relationship was analyzed. The information obtained from these calculations is very important for quantitative analyses and is highly useful to the understanding of the binding mechanism. Figure 1 shows the inhibitor-residue interaction spectra calculated by the free energy decomposition, which suggests that the interaction spectra of INHs 1-8 with the exosite of BACE1 are closely related and reflect their similar binding modes.

From these results, we considered prudently to include in the reduced model not just those amino acids involved in the most relevant MI displayed in the different spectra, but also all the residues involved in stabilizing and destabilizing interactions showing nonnegligible contribution in the per residue energy decomposition spectra. Thus, residues Glu163, Glu255, Lys256, Phe257, Pro258, Asp259, Gly260, Phe261, Trp262, Leu263, Gly264, Glu265, Gln266, Leu267, Val268, Cys269, Trp270, Gln271, Ala272, Gly273, Thr274, Asp311, Val312, Ala313, Thr314, Ser315, Gln316, Asp317, Asp318, Cys319, Tyr320, Lys321, and Phe322 were included in the reduced model for the exosite of BACE1, and, therefore, a final number of 33 amino acids were included in our model.

\subsubsection{Quantum mechanics calculations}

Considering the 33 amino acids selected on the basis of the per-residue energy decomposition spectra plus the residues from the inhibitor, the number of atoms of the reduced model rises up to 718 in BACE1-INH 8 complex. The size of the molecular system and the complexity of the structures under investigation restricted the choice of the quantum mechanical method to be used. Consequently, geometrical optimizations for the different complexes were carried out at semiempirical level (PM6 and PM6-COSMOS), whereas single point calculation at DFT (B3LYP/6-31G(d) levels were performed in order to obtain the energy values of the complexes under study.

The binding energy of the complexes at quantum mechanical level was calculated using the supermolecular approach, that is, calculated by subtracting the energies of the isolated compounds (in the complex geometry) from the energy of BACE1-INH complex.

$$
\mathrm{BE}_{\mathrm{QM}}=E_{\mathrm{Exo}+\mathrm{INH}}-\left(E_{\mathrm{Exo}}+E_{\mathrm{INH}}\right)
$$

where $\mathrm{BE}_{\mathrm{QM}}$ is the binding energy at quantum mechanical level, $E_{\mathrm{Exo}}+\mathrm{INH}$ the exosite-inhibitor complex energy, $E_{\text {Exo }}$ the energy of the reduced receptor model (exosite), and $E_{\mathrm{INH}}$ the energy of the inhibitor.

The PM6 and PM6-COSMOS calculations were carried out using MOPAC program (James Stewart, 2012), whereas DFT calculations were performed by using Gaussian 09 software (Frisch et al., 2009).

\subsubsection{Topological study of the electron charge density distribution}

For the study of MI between peptides $\mathbf{1 - 8}$ and the BACE1-exosite, the molecular complexes obtained for our "reduced model system" were used as input for the calculation of the charge density. The complexity of the system under study restricts the choice of the quantum mechanical method to be used. Therefore, single point calculations were performed with the Gaussian 09 program package employing the B3LYP hybrid functional and $6-31 \mathrm{G}(\mathrm{d})$ as a basis set. This type of calculation has been used in recent works on the topology of the charge density $\left(\rho_{(r)}\right)$ because it ensures a reasonable compromise between the wave function quality required to obtain reliable values of the derivatives of $\rho_{(r)}$ and the computer power available (Tosso et al., 2013). The topological properties of a scalar field such as $\rho_{(r)}$ are summarized in terms of their critical points, i.e., the points rc where $\nabla \rho_{(r)}=0$. Critical points are classified according to their type $(\omega, \sigma)$ by stating their rank, $\omega$ and signature, $\sigma$. The rank is equal to the number of nonzero eigenvalues of the Hessian matrix of $\rho_{(r)}$ at $(\mathrm{rc})$, while the signature is the algebraic sum of the signs of the eigenvalues of this matrix. Critical points of $(3,-1)$ and $(3,+1)$ type describe saddle points, while the $(3,-3)$ is the maximum and $(3$, $+3)$ is the minimum in the field. Among these critical points, the $(3,-1)$ or bond critical points (BCPs) are the most relevant ones since they are found between any two atoms linked by a chemical bond. The determination of all the bond BCPs and the corresponding bond paths that connect these points with the bonded nuclei, the calculations of local topological properties of $\rho_{(r)}$ at the BCPs, as well as, the display of molecular graphs were performed with the AIMAll software (Keith, 2012). 


\begin{tabular}{|lll|}
\hline TBSD 1145143 & Initial & CE: PS \\
8 February 2016 & CS \\
\hline
\end{tabular}

(a)

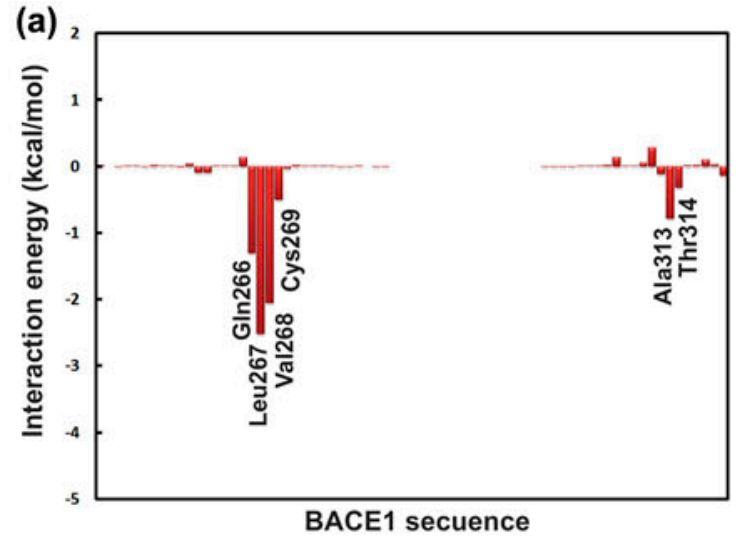

(c) 2
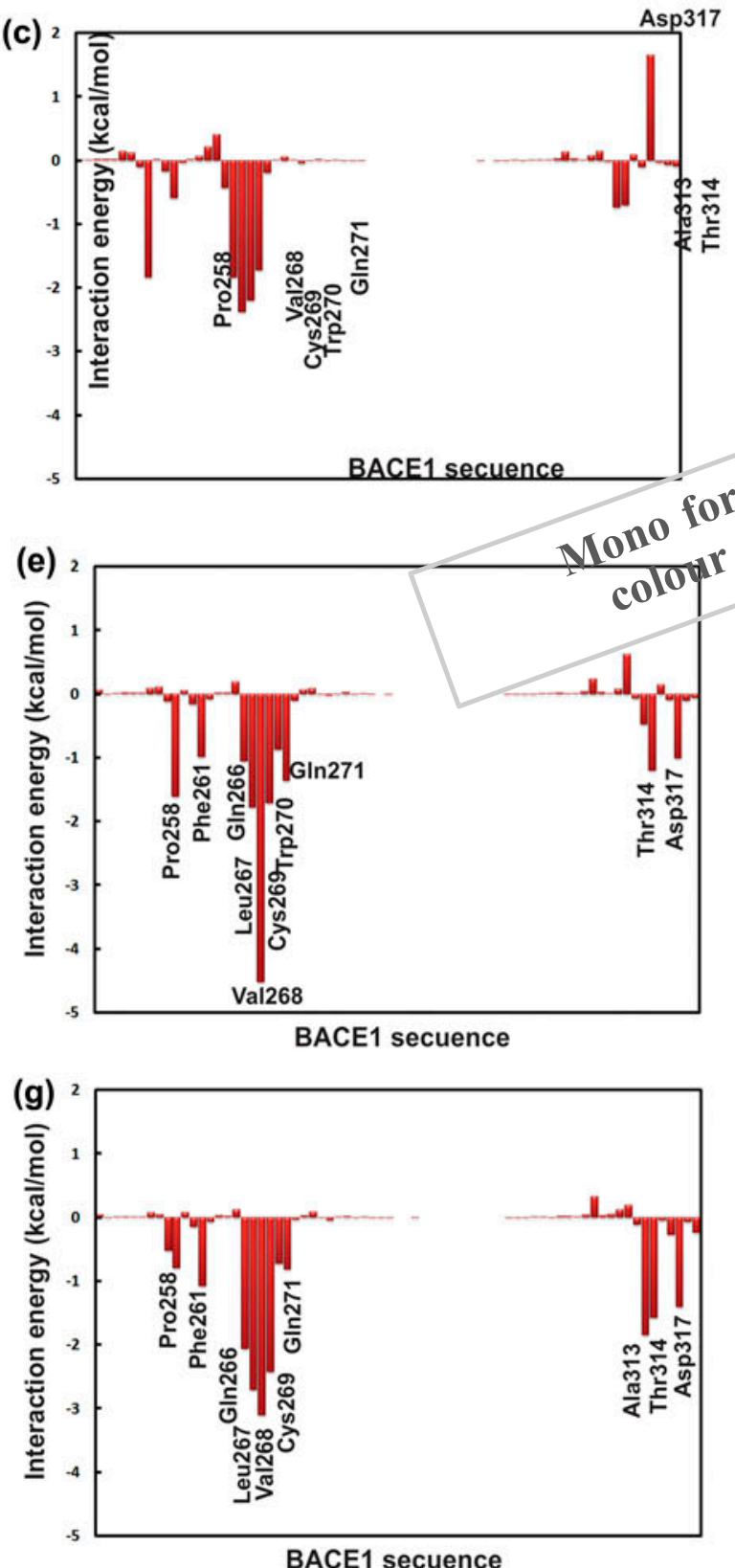

(b)

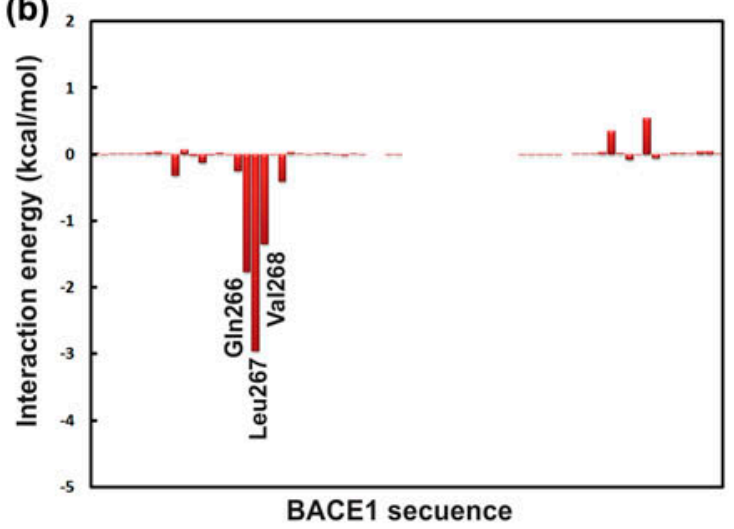

(d)

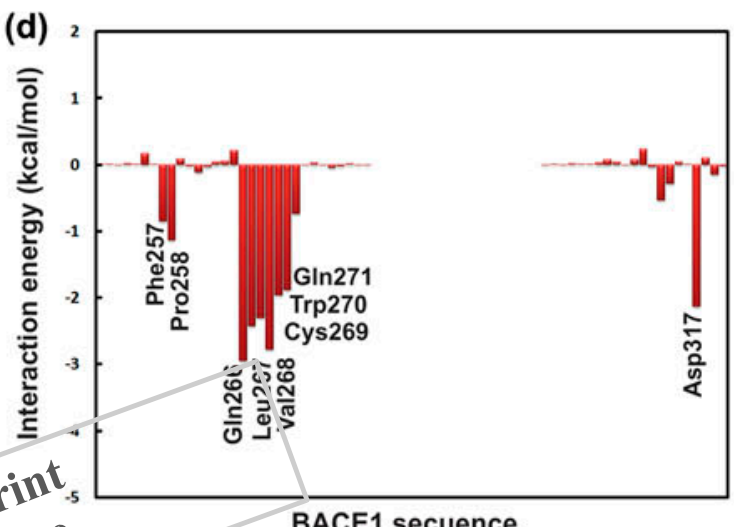

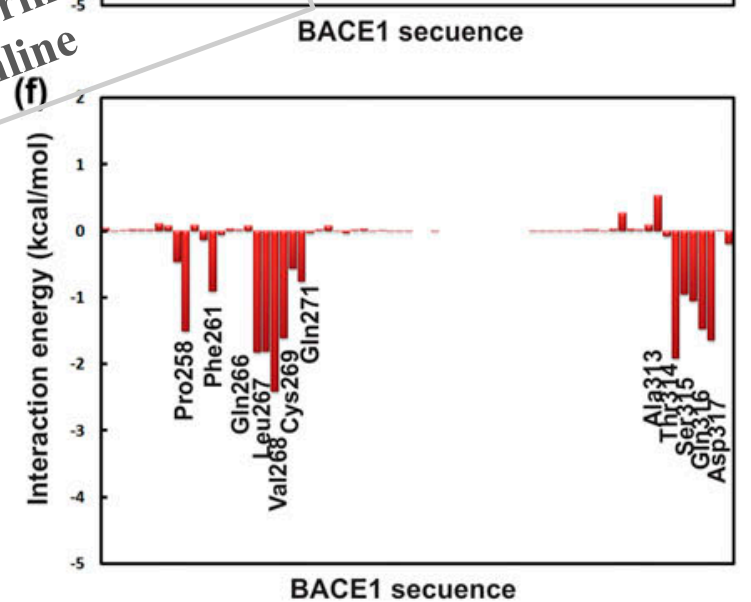

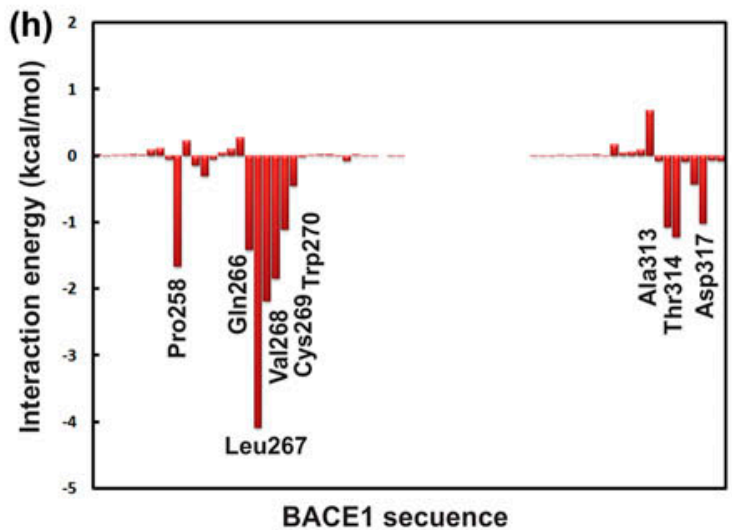

Figure 1. Spectra of residue interactions obtained for the complexes Inhibitor-BACE1: (a) 1, (b) 2, (c) 3, (d) 4, (e) 5, (f) 6, (g) 7, and (h) 8 according to the MM-GBSA method.

Note: The $x$-axis denotes the residue number of BACE1 and the $y$-axis denotes the interaction energy between the inhibitor and specific residues. 
It should be noted that QTAIM calculations were carried out using a higher level of computations than that used for geometrical optimization. Thus, from the point of view of the DFT level applied, the PM6-minimized structure is a "random conformation," and some especially weaker bonds cannot be correctly identified. Certainly the ideal situation would be to perform structure minimizations at the same computational level than the QTAIM analysis. However, due to the computational cost of performing an energy minimization at the DFT level, it would be necessary to reduce the size of the model system, losing the additive part of the intermolecular interactions (cooperative effects). Therefore, we have preferred to resign some quality and build a reduced model as representative as possible of the active site. The argument is that the QTAIM methodology is relatively insensitive to the method of calculation (Castillo \& Boyd, 2005; Jabłoński \& Palusiak, 2010; Matta, 2010). Therefore, the topological elements which are present at the higher level wave function, though more accurately computed will be essentially the same than in the lower level wave function, provided that the geometry is the same.

\section{Results and discussion}

\subsection{Equilibrium of the molecular dynamics simulation}

The root-mean-square deviation (RMSD) of each snapshot relative to the initial structure was calculated to monitor the stability of each trajectory. The RMSD obtained for the backbone atoms for each component of complex (BACE1 and INH chains) of all the trajectories remains constant during the last $5 \mathrm{~ns}$ of MD simulation, ures $3 \mathrm{~S}$ and $4 \mathrm{~S}$ and Table $1 \mathrm{~S}$ in supplementary material). The trajectory of last $5 \mathrm{~ns}$ of each replicate was taken for the structural and energetic analysis.

\subsection{Binding free energy}

By the MM-GBSA analysis (Kollman et al., 2000), the total free energy of binding might be separated into electrostatic, van der Waals, and solute-solvent interactions, thus gaining additional insights into the physics of the BACE1-INH association process. The binding free energy and the energy components of the complexes are summarized in Table 2. According to Table 2, electrostatic $\left(\Delta E_{\text {ele }}\right)$, van der Waals $\left(\Delta E_{\mathrm{vdw}}\right)$, and terms of nonpolar solvation energies $\left(\Delta G_{\mathrm{NP}}\right)$ provide the major favorable contributions to the INH binding, whereas polar solvation energies $\left(\Delta G_{\mathrm{GB}}\right)$ impair to the INH binding. Further insight into the forces involved in BACE1INH complex formation can be obtained by analyzing the electrostatic $\left(\Delta G_{\text {ele,tot }}\right)$ and nonelectrostatic $\left(\Delta G_{\mathrm{NP}, \text { tot }}\right)$ contributions (Table 2). Indeed, from Table 2, we can appreciate that, despite the favorable electrostatic energies in the gas phase $\left(\Delta E_{\text {ele }}\right)$, the contributions of polar solvation energies to binding $\left(\Delta G_{\mathrm{GB}}\right)$ are unfavorable for all the complexes studied here, and the $\Delta G_{\text {ele,tot }}$ (the sum of $\Delta E_{\text {ele }}$ and $\left.\Delta G_{\mathrm{GB}}\right)$ does not favor the binding. Table 2 also suggests that the net result of nonelectrostatic interaction $\left(\Delta G_{\mathrm{NP}, \text { tot }}\right.$, the sum of $\Delta E_{\mathrm{vdw}}$ and $\left.\Delta G_{\mathrm{NP}}\right)$ is favorable for the formation of all the complexes. It should be noted that this behavior has been proposed previously as a general trend for noncovalent ligand-receptor associations (Chen, Yang, Yi, Shi, \& Zhang, 2009). From the above results, we can conclude that the binding free energies obtained for these complexes are driven by more favorable nonpolar interactions rather than by electrostatic ones.

Next, the binding energies $\left(\mathrm{BE}=\Delta G_{\text {bind }}\right)$ obtained for the different complexes were evaluated. The correlation coefficient obtained between the experimental percentages of inhibition vs. the binding energies calculated from MM-GBSA was acceptable giving a value of $r=-.86$ (Figure 2(a)). On the basis of the binding energies obtained from our MD simulations, a good inhibitor

Table 2. Binding free energies computed by the MM-GBSA method (kcal/mol).

\begin{tabular}{lccccccrr}
\hline INH & $\Delta E_{\text {ele }}$ & $\Delta E_{\mathrm{vdw}}$ & $\Delta G_{\mathrm{GB}}$ & $\Delta G_{\mathrm{NP}}$ & $\Delta G_{\text {ele,tot }}$ & $\Delta G_{\mathrm{NP}, \text { tot }}$ & $\Delta G_{\text {Bind }}$ & $\%$ inhibition \\
\hline 1 & -9.16 & -22.36 & 20.49 & -3.49 & 11.33 & -25.85 & -14.51 \\
2 & -8.18 & -25.57 & 22.42 & -4.76 & 14.24 & -30.34 & -16.09 & 2.50 \\
3 & -6.99 & -42.66 & 30.12 & -6.50 & 23.13 & -49.16 & -26.03 & 27.00 \\
4 & -40.59 & -43.77 & 55.56 & -6.35 & 14.97 & -50.13 & -35.16 & 37.00 \\
5 & -29.79 & -53.77 & 57.05 & -7.38 & 27.26 & -61.15 & -33.89 & 65.00 \\
6 & -58.33 & -57.56 & 80.27 & -8.14 & 21.93 & -65.70 & -43.76 & 67.00 \\
7 & -29.75 & -64.26 & 57.66 & -8.38 & 27.91 & -72.64 & -44.73 & 70.00 \\
8 & -3.07 & -46.29 & 26.38 & -6.76 & 23.30 & -53.05 & -29.75 & 71.00 \\
9 & -130.71 & -39.58 & 156.68 & -5.90 & 25.97 & -33.68 & -19.51 & $20.70^{\mathrm{b}}$ \\
10 & -92.71 & -52.53 & 119.28 & -7.76 & 26.27 & -44.77 & -33.72 & $33.10^{\mathrm{b}}$ \\
\hline
\end{tabular}

${ }^{\mathrm{a}} \%$ inhibition was taken from reference Kornacker et al. (2005).

$\mathrm{b}_{\%}$ inhibition obtained from ours experimental results. 
(a)

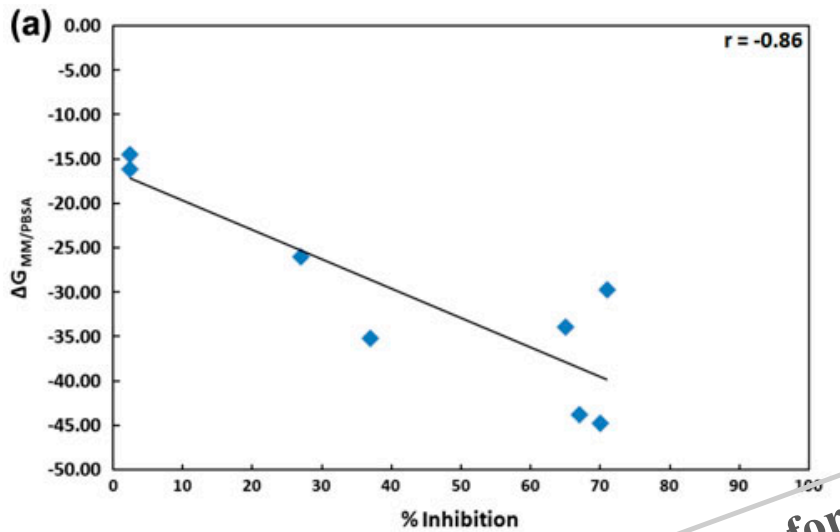

(b)

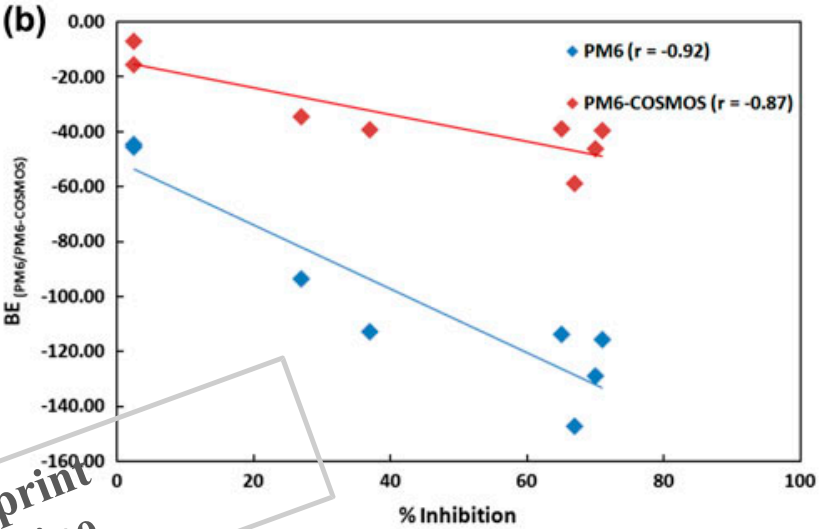

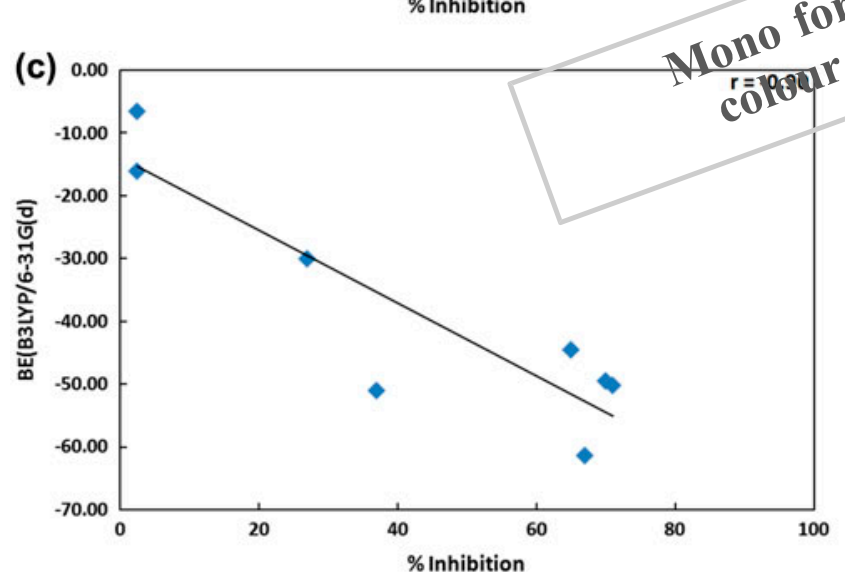
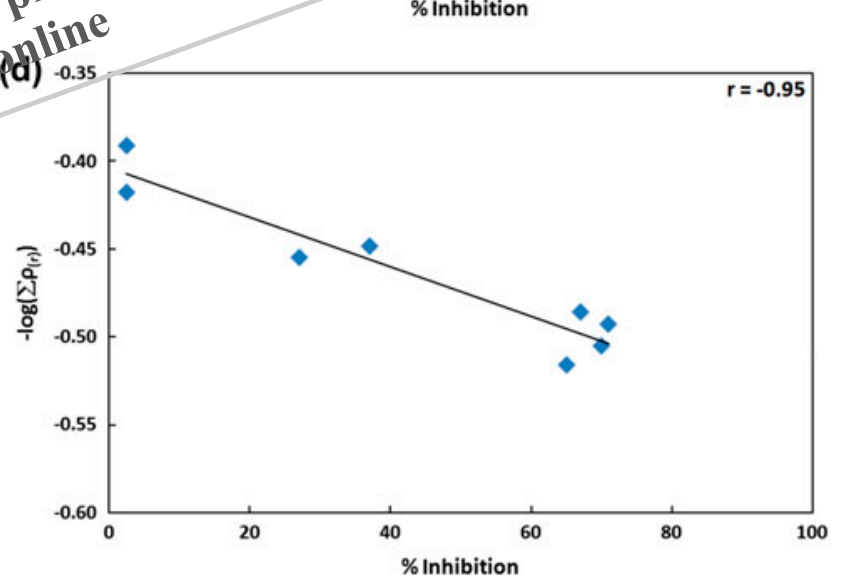

Figure 2. Correlations obtained between the experimental percentages of inhibition vs. the binding energies calculated from (a) MM-GBSA, (b) PM6 (light blue) and PM6-COSMOS (red), (c) B3LYP/6-31G(d), and (d) $-\log \left(\sum \rho_{(r)}\right)$ calculations.

Note: It should be noted that the values of $\sum \rho_{(r)}$ have been plotted as -log in order to obtain a negative slope for comparative purposes.

can be differentiated from a very weak inhibitor $(-44.73 \mathrm{kcal} / \mathrm{mol}$ for compound $7 \mathrm{vs}$. $-14.51 \mathrm{kcal} / \mathrm{mol}$ for compound 1), but peptides with similar binding affinities cannot be easily differentiated. It is interesting to note that compound $\mathbf{8}$ possesses the strongest inhibitory effect; however, it has a relatively high $\mathrm{BE}$ $(-29.75 \mathrm{kcal} / \mathrm{mol})$.

It should be noted that MD simulations might neglect or poorly approximate terms that might be playing determinant roles such as lone pair directionality in hydrogen bonds, explicit $\pi-\pi$ stacking polarization effects, hydrogen bonding networks, induced fit, and conformational entropy. Thus, we cannot expect to obtain clear differences between compounds possessing relatively similar binding energies. At this stage of our work, we consider the trend predicted by the MD simulations as indicative and certainly interesting for an exploratory analysis, but on the other hand, the approximations involved in this approach compel us to go beyond to the classical treatment of the interactions in order to confirm our results. Thus, in the next step, the reduced model systems were optimized using the PM6, PM6-COSMOS semiempirical methods, combined with DFT single point calculations.

\subsection{Ouantum mechanics calculations}

The starting geometries for each complex were obtained from the coordinates of the conformations displaying the lowest potential energies during the simulations. PM6 optimizations were performed considering all amino acids (included in the reduced model, see methods of calculations section). Next, in order to consider the solvent effects, PM6-COSMOS optimizations were carried out for the different complexes. Finally, DFT (B3YP/6$31 \mathrm{G}(\mathrm{d})$ ) single point calculation were carried out for each complex optimized from PM6 computations.

The RMSD data for the results obtained from PM6 and PM6-COSMOS are summarized in Table 3. From these results, it is clear that the inclusion of the COSMO continuum solvent model causes no geometry changes from the results generated by PM6 (RMSD $<1.5 \AA$ ), at least for the complexes under study. 
Table 3. Structural difference between the optimized geometries at PM6 and PM6-COSMOS level.

\begin{tabular}{lccc}
\hline Complex & Exosite + Ligand & Exosite & Ligand \\
\hline $\mathbf{1}$ & 1 & 1.02 & .73 \\
$\mathbf{2}$ & 1.28 & 1.35 & .62 \\
$\mathbf{3}$ & .98 & 1.08 & .67 \\
$\mathbf{4}$ & 1.07 & 1.15 & .45 \\
$\mathbf{5}$ & 1.37 & 1.53 & .46 \\
$\mathbf{6}$ & .86 & .97 & .31 \\
$\mathbf{7}$ & .8 & .9 & .32 \\
$\mathbf{8}$ & .87 & .97 & .22 \\
\hline
\end{tabular}

Notes: Root-mean-square deviation (RMSD) was calculated using UCSF Chimera program.

Once the BE of the different complexes were obtained from the theoretical calculations (see Table 2S), we calculate the different correlations between the theoretical calculations and the experimental data (percentage of inhibition) reported in reference (Kornacker et al., 2005).

Figure 2(b) and (c) gives a graphical representation of the BE obtained from PM6 and DFT (B3LYP/6-31G (d)) calculations vs. the experimental data. The figure displays the following correlation coefficients $r=-.92$, -.87 , and -.90, using PM6, PM6-COSMOS, and B3LYP/6-31G(d)) calculations, respectively. These results are satisfactory, considering the type of approximation used. From these results, it appears that the predicted first-principle structure of the primary binding pocket of BACE1-exosite leads to correct predictions of the critical residues for binding INHs and gives relative binding affinities that correlate fairly well with the experimental data. (Kornacker et al., 2005)

PM6 and DFT calculations performed here may not properly consider the dispersion interactions. Fortunately, in this case, it appears that such limitations are not severe enough to prevent us from obtaining our goals. Such an assumption appears to be reasonable, considering the significant correlation obtained between the experimental data and the theoretical calculations performed. However, a kind of error-cancelation might have taken place, in which case the approaches used in this study might be operative only for the INHs analyzed here. An additional validation and more calculations might be required to extend these approaches to other compounds possessing different structures. It is clear that more accurate calculations, such as QTAIM (Bader, 1985) analysis, are necessary for a detailed description of these interactions. Such calculations are presented in the next section.

\subsection{Evaluating the MI for the different complexes using QTAIM calculations}

The topological analysis of the electron density distribution is widely applied to the characterization of the inter- bonds in small gas-phase complexes (by means of the QTAIM methodology). While this theory has traditionally belonged to the field of the theoretical chemists, there are a number of recent works where it has been applied to the study of large biomolecular complexes providing a very detailed description of the binding event (Andujar et al., 2012; Angelina, Andujar, Tosso, Enriz, \& Peruchena, 2014; Párraga et al., 2013; Tosso et al., 2013), therefore suggesting that this theory should be also adopted by the medicinal chemists and molecular modelers. Accordingly, we performed a QTAIM description of the interactions on the reduced models of the BACE1-INH complexes.

Previous to the QTAIM analysis, some comments about the selected peptides must be done. As can be seen in Table 1, the small-size peptides selected (INH 1-8) contain from 3 to 10 amino acids. The INH 7 represents the entire peptide sequence $\left(\mathrm{Ac}-\mathrm{Thr}_{3}-\mathrm{Thr}_{4}-\mathrm{Tyr}_{5}-\mathrm{Pro}_{6}-\right.$ $\mathrm{Tyr}_{7}-\mathrm{Phe}_{8}-\mathrm{Ile}_{9}-\mathrm{Pro}_{10}-\mathrm{Leu}_{17}-\mathrm{Pro}_{12}-\mathrm{NH}_{2}$ ), and the other peptides lack some of the residues either in the Ac-terminal or $\mathrm{NH}_{2}$-terminal fragment. By inspecting the inhibition data in Table 2, one might infer that the $-\mathrm{Tyr}_{5}-\mathrm{Pro}_{6}-$ sequence near to the Ac-terminal is essential for activity because INHs 1 and $\mathbf{2}$ which lack these residues show negligible inhibitory activity against BACE1. Moreover, INHs 3 and $\mathbf{4}$ which lack some residues from the $\mathrm{NH}_{2}-$ terminal fragment displayed just the half of the activity of INHs 6 to 8. Pro 10 and $\mathrm{Leu}_{11}$ residues are present in the most active peptides, but they are not present in INH 3 and INH $4\left(\mathrm{Pro}_{10}\right.$ and $\mathrm{Leu}_{11}$ in INH 3 and Leul1 in INH 4) indicating that they are important for the modulatory activity as well. On the contrary, the absence of the $\mathrm{Thr}_{3}-\mathrm{Thr}_{4}$ sequence $/ \mathrm{Pro}_{12}$ residue from the $\mathrm{C}-\mathrm{N}$ - terminus of INH 7, respectively, does not seem to affect the activity of the peptide since these terminal residues are missing in INH 8 which is the most active compound in this series.

Figure 5 shows the complexes obtained for INHs 1, $\mathbf{3}$, and $\mathbf{8}$ interacting with the BACE1-exosite, which are representative reduced models for poor, intermediate, and good inhibitory activities, respectively (Figure 3 ).

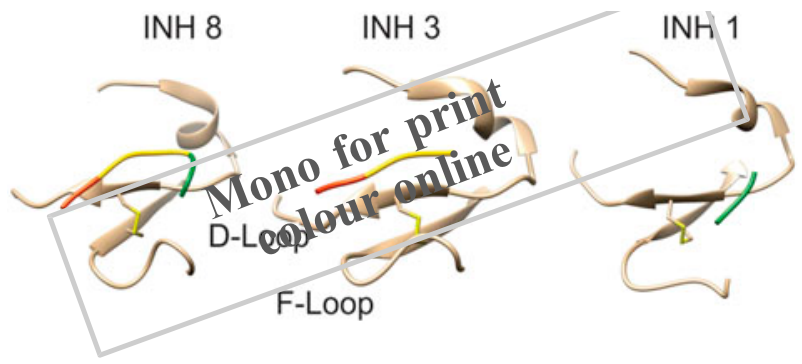

Figure 3. Spatial view of INHs $\mathbf{1}, \mathbf{3}$, and $\mathbf{8}$ in the exosite. Note: Acetyl-terminal fragment (in red), $\mathrm{NH}_{2}$-terminal fragment (in green), and the residues connecting both end fragments (in yellow). 
It can be seen that the same residues of the inhibitor (i.e., peptide fragments with the same color) bind approximately to the same site in the BACE1-exosite, in the three complexes. Thus, for example, the Ac- $\mathrm{Tyr}_{5^{-}}$ $\mathrm{Pro}_{6}$ - pattern (in orange) is interacting with residues from the D-loop and F-loop in INHs 3 and 8. Therefore, according to the previous observations, one might anticipate that the interactions of $\mathrm{Ac}^{-} \mathrm{Tyr}_{5}-\mathrm{Pro}_{6}{ }^{-}$end with specific residues from these loops of the BACE1 would be, at least partially, responsible for the modulatory activity of these peptides.

At this point, it is interesting to estimate the strength of the network of noncovalent interactions established

15 between the BACE1-exosite and the small peptides selected. Starting with strong and moderate hydrogen bonds, moving on to weaker polar interactions, and ending with stacking and T-shape-like interactions, all of them can be investigated within the framework of the density functional theory and the QTAIM. Figure 2(d) shows the correlation obtained between the experimental percentages of inhibition values (\%inhibition) vs. $-\log$ $\left(\sum \rho_{(r)}\right)$ corresponding to the BCPs of inter- and intramolecular interactions in (BACE1-INH) complex.

The correlation coefficient $(r=-.95)$ is better than those obtained from the BE calculated at PM6, PM6COSMOS, and DFT levels of theory (Figure 2(b) and (c), respectively). Also, it should be noted that the correlation obtained using the $\sum \rho_{(r)}$ is significantly better than that obtained from binding energies calculated from MM-GBSA (Figure 2(a)). Moreover, the advantage of the charge density sum over the BE is that it can be partitioned into several contributions that account for the anchoring strength of each residue or sequence of resi-

35 dues into the INH. In addition, the standard deviation of regression (S_reg) (also, namely residual standard deviation or standard error of estimate) is an absolute measure of dispersion of the $Y$ values around the regression line, and it can be used as a measure of the closeness of the

40 relationship between the variables. The smaller the S_reg, closer the relationship; the higher the value of S_reg, the more the actual observation tend to scatter away from the regression line. In other words, the range within which the S_reg must lie is as close to zero, 45 denoting an excellent relationship between the considered variables (Riu \& Rius, 1996; William, Saul, William, \& Brian, 1986). The residual standard deviations in Table $2 \mathrm{~S}$ are of different units in the different cases, and therefore cannot be compared directly. Thus,

50 the residual standard deviations were divided by the slope of the fitting line to be converted into the same units (last row in Table 2S). It is interesting to note that the S_reg obtained for the correlation using $\sum \rho_{(r)}$ displayed lower values of $S_{-}$reg in comparison with those obtained for the rest of correlations (see Tab̂le $2 \mathrm{~S}$ in supplementary material).
Accordingly, Figure 4 shows the sum of the charge density values at the BCPs due to inter- and intra-interactions in BACE1-INH complexes. It is partitioned into three contributions corresponding to the Ac-terminal fragment (Ac- $\mathrm{Thr}_{3}-\mathrm{Thr}_{4}-\mathrm{Tyr}_{5}-\mathrm{Pro}_{6}-$ in red color); the $\mathrm{NH}_{2}$-terminal fragment $\left(-\mathrm{Pro}_{10}-\mathrm{Leu}_{11}-\mathrm{Pro}_{12}-\mathrm{NH}_{2}\right.$ in green), and the connector fragment $\left(-\mathrm{Tyr}_{7}-\mathrm{Phe}_{8}-\mathrm{Ile}_{9}-\right.$ in yellow) between the two previous one. Note that one or more of these residues are absent in INHs other than INH 7 (see Table 1).

Each category of the stacked bars indicates the anchoring strength of a different fragment of the inhibitor sequence, whereas the total height of the stacked bars indicates the binding strength of the entire peptide.

Since INHs 1 and $\mathbf{2}$ lack the Ac-terminal fragment (Ac- $\mathrm{Thr}_{3}-\mathrm{Thr}_{4}-\mathrm{Tyr}_{5}-\mathrm{Pro}_{6-}$ ), which apparently is crucial for modulatory activity, from now the discussion is centered in INHs $\mathbf{3}$ to $\mathbf{8}$.

\subsection{Interactions due to the residues of the Ac- terminal fragment}

Figure 5 show the interactions of the INHs $\mathbf{3}$ to 8 , respectively, within the BACE1-exosite framework.

By the comparison of these figures, it can be seen that the peptide fragment $A c-\mathrm{Tyr}_{5}-\mathrm{Pro}_{6}$ - from INHs 4 (Figure 5(b)) as well as from INH 8 (Figure 5(f)) is inserted between the backbones of the D- and F-loops. In both complexes, the loops are farther apart from each other than in the remaining complexes, and the acetyl group is interacting with the backbone of $\mathrm{Ala}_{272}$ and the side chain of $\mathrm{Glu}_{317}$. In fact, the $\mathrm{C}_{\alpha}-\mathrm{C}_{\alpha}$ separation distance between $\mathrm{Gln}_{271}$ (D-loop) and $\mathrm{Asp}_{317}$ (F-loop) is remarkably larger when BACE1 is complexed with INH

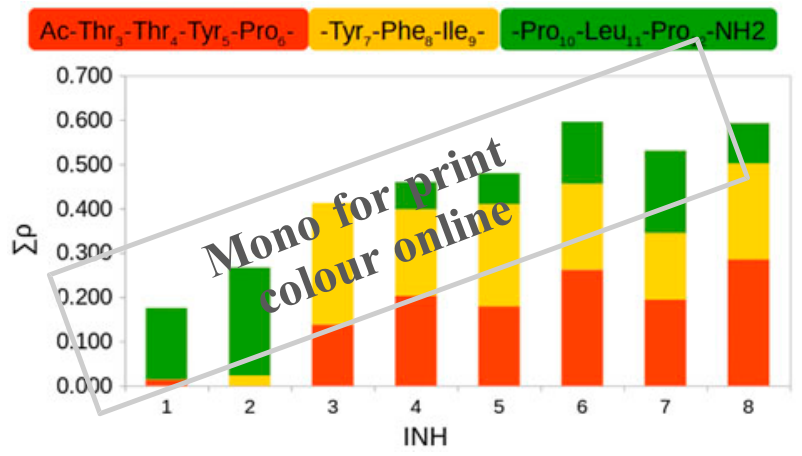

Figure 4. Charge density sum values at the $\operatorname{BCPs}\left(\sum \rho_{(r)}\right)$, due to inter and intra-molecular interactions in BACE1-INH complexes.

Notes: These values were partitioned into three contributions: (in red) due to interactions involving residues of the acetyl-terminal fragment; (in green) involving residues of the $\mathrm{NH}_{2}$-terminal fragment; and interactions involving residues connecting both end fragments, in yellow. 

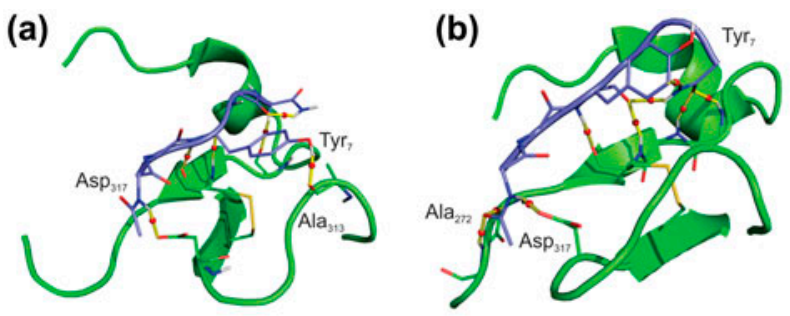

(c)

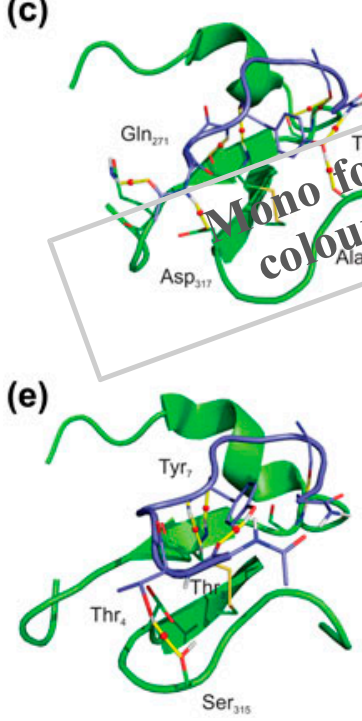

(d)

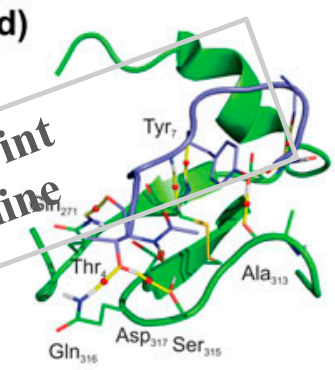

(f)

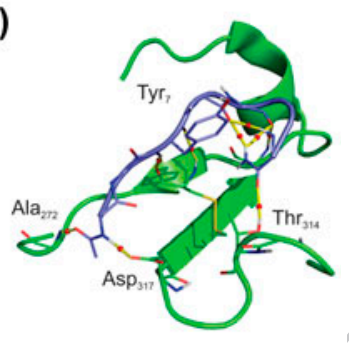

Figure 5. Noncovalent interactions for the different complexes (a) 3, (b) 4, (c) 5, (d) 6, (e) 7, and (f) 8 .

Notes: The inhibitors are shown in blue and BACE1-exosite in green. Also the elements of the topology of the electron density are shown: yellow sticks represent the bond paths connecting the nuclei and the red circles on them are the bond critical points $(3,-1$ critical points). Due to the complexity of the structure, only the most relevant interactions are shown in this figure.

$4(6.956 \AA)$ and INH $8(8,055 \AA)$ than with INH 3 (4.943 $\AA)$, INH 6 (4.901 $\AA), \operatorname{INH} 7$ (4.852 $\AA)$, and INH 5 (4.613 $\AA$ ).

In complexes of BACE1 with INHs $\mathbf{7 , 6}, \mathbf{5}$, and $\mathbf{3}$, because the loops are closer together, the Ac-terminal peptide fragment cannot be inserted between them but instead, it just lies over F-and/or D-loops, interacting with side chains of one or both loops.

Going back to Figure 4, it can be seen in this figure that the acetyl end from INH $\mathbf{8}$ is more strongly anchored into the exosite than the remaining peptides. Besides INH 8, the second strongest anchored peptide is INH 6. INHs 6 and 7 possess one and two additional threonine residues in their Ac-terminal peptide fragment, respectively, as compared with the other peptides. Thus, instead of comparing the absolute anchoring strength of the Ac-terminal peptide fragment in the different complexes, it is more appropriate to measure how "efficient" is that binding to compute the anchoring strength per number of atoms of the Ac-terminal peptide fragment. Figure 6 shows the sum of the charge density values corresponding to the interactions of the Ac-terminal peptide fragment within the exosite divided its number of atoms, $\sum \rho /$ NA.

The $\sum \rho * / \mathrm{NA}$ ratio clearly shows that the Ac-terminal peptide fragment from INHs $\mathbf{4}$ and $\mathbf{8}$ binds more efficiently to the exosite than in the same fragment from INHs 3, 5, 6, and 7. This finding is in agreement with the fact that in the first two complexes, the acetyl end is deeply inserted between the two loops interacting with residues from both loops while in the last four complexes they just lie over the F- and/or D-loops.

The next question one may ask is what makes the D- and F-loops be farther apart (and therefore the acetyl end more inserted between both loops) in some complexes than in the others. We explore this question in the next section.

\subsection{Interactions of the connector fragment residues}

Among the residues of the connector fragment of the $\mathrm{INHs}, \mathrm{Tyr}_{7}$ shows significant differences in its interaction pattern. By inspecting the complexes of BACE1 with the INHs (Figures 5(a)-(f)), it can be seen that in those complexes where the D- and F-loops are closer together (INHs 3, 5, and 6), the $\mathrm{Tyr}_{7}$ residue forms an H-bond with the backbone carbonyl of $\mathrm{Ala}_{313}$ (i.e, $\left(\mathrm{Ala}_{313}\right.$ ) $\left.\mathrm{O} \cdot \cdots \mathrm{H}-\mathrm{O}\left(\mathrm{Tyr}_{7}\right)\right)$, whereas in complexes where both loops are farther apart (INHs 4 and 8), the $\left(\mathrm{Ala}_{313}\right) \mathrm{O} \cdots \mathrm{H}-\mathrm{O}$ $\left(\mathrm{Tyr}_{7}\right)$ H-bond is absent and $\mathrm{Tyr}_{7}$ adopts a different conformation. Both situations can be compared in Figure 7. Figure 7 shows the conformational changes experienced by the backbone of the exosite after binding to INH $\mathbf{5}$ (orange) wherein the $\left(\mathrm{Ala}_{313}\right) \mathrm{O} \cdot \cdot \mathrm{H}-\mathrm{O}\left(\mathrm{Tyr}_{7}\right)$ is present, and also to INH 8 (green) wherein this H-bond is absent.

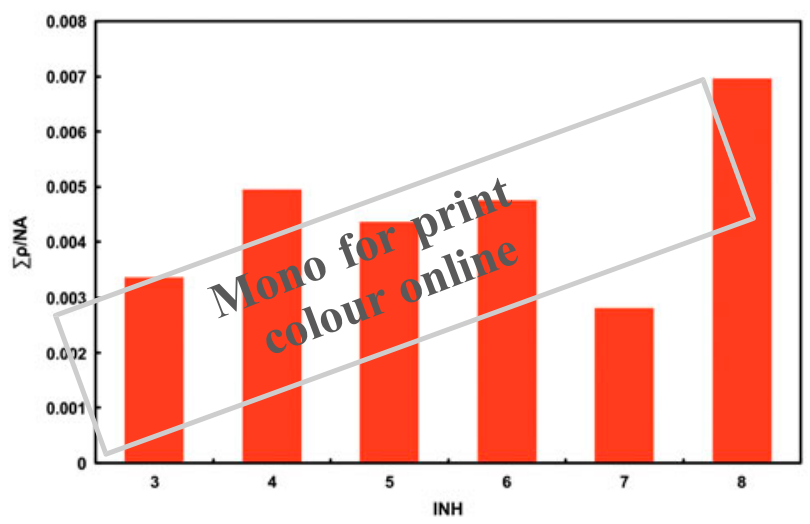

Figure 6. Charge density sum values/number of atoms ratio obtained for the intermolecular interactions for those inhibitors possessing the Ac end fragment, $\left(\sum \rho / \mathrm{NA}(\mathrm{Ac}-\mathrm{TTYP})\right)$ in selected BACE1-INH complexes. 


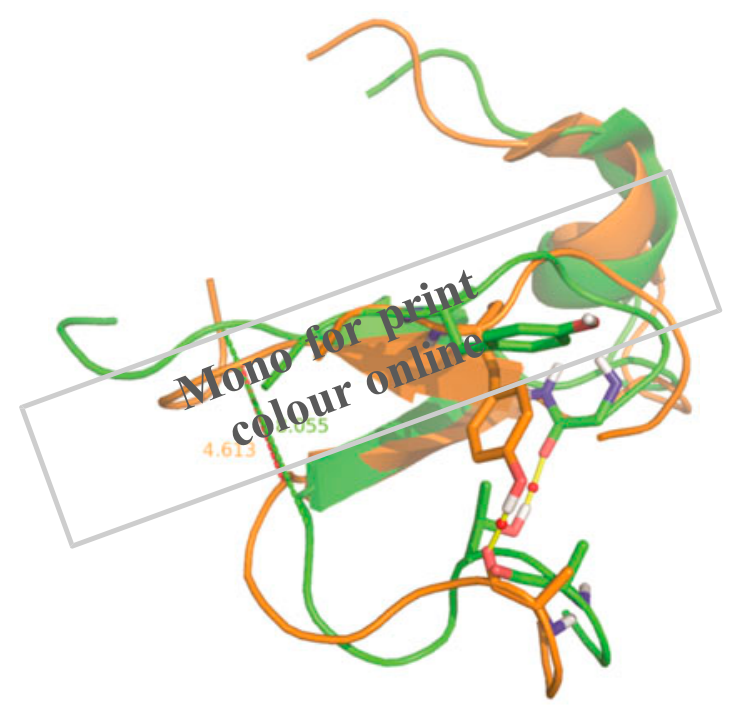

Figure 7. Backbone alignment for complexes BACE1-INH 8 (green) and BACE1-INH 5 (orange).

Notes: The figure shows the interactions behind the conformational changes of the D- and F-loops. Also the $\mathrm{C}_{\alpha}-\mathrm{C}_{\alpha}$ separation distance (in $\AA$ ) between $\mathrm{Gln}_{271}$ (D-loop) and $\mathrm{Asp}_{317}$ (Floop) is depicted for both complexes.

It is clearly seen in this figure that in complex BACE1-INH 5 complex the interaction of $\mathrm{Tyr}_{7}$ with $\mathrm{Ala}_{313}$ might help to keep the $\mathrm{D}$ - and F-loops close together. On the contrary, in the complex of INH $\mathbf{8}$, the $\mathrm{NH}_{2}$-terminal peptide fragment forms an $\mathrm{H}$-bond with $\mathrm{Thr}_{314}$ which might help to keep the F-loop and D-loop more separated from each other.

But why $\left(\mathrm{Ala}_{313}\right) \mathrm{O} \cdots \mathrm{H}-\mathrm{O}\left(\mathrm{Tyr}_{7}\right) \mathrm{H}$-bond might be formed in some complexes but not in the others? The answer might lie in the sequence differences in the $\mathrm{NH}_{2}-$ terminal peptide fragment.

\subsection{Interactions due to the residues of the $\mathrm{NH}_{2-}$ terminal fragment}

15 By inspecting the complexes of BACE1 with the different INHs (Figure 5(a)-(f)), it can be seen that in INHs 5 and 6 which have the entire sequence $-\mathrm{Pro}_{10^{-}} \mathrm{Leu}_{11^{-}}$ $\mathrm{Pro}_{12^{-}}$in the $\mathrm{NH}_{2}$-terminal peptide fragment, the $\mathrm{NH}_{2}$ group is properly placed to act as H-donor in a bifurcated H-bond with the oxygen atom of $\mathrm{Tyr}_{7}$, enhancing the $\left(\mathrm{Ala}_{313}\right) \mathrm{O} \cdots \mathrm{H}-\mathrm{O}\left(\mathrm{Tyr}_{7}\right) \mathrm{H}$-bond (see Figure 5(c) and (d)). In the case of INH 7, even when it has the full sequence at $\mathrm{NH}_{2}$-terminal peptide fragment, the $\left(\mathrm{Ala}_{313}\right)$ $\mathrm{O} \cdots \mathrm{H}-\mathrm{O}\left(\mathrm{Tyr}_{7}\right) \mathrm{H}$-bond is absent due to the formation of an intramolecular H-bond with the backbone of $\mathrm{Thr}_{3}$.

On the other hand, INHs 4 and 8 lack the $\mathrm{Leu}_{11^{-}}$ Pro12- and $\mathrm{Pro}_{12}$ - residues in the $\mathrm{NH}_{2}$-terminal fragment, respectively, and hence the terminal $\mathrm{NH}_{2}$ group is not properly placed to stabilize the $\left(\mathrm{Ala}_{313}\right) \mathrm{O} \cdots \mathrm{H}-\mathrm{O}\left(\mathrm{Tyr}_{7}\right)$
H-bond. As was discussed above, in the complex of
BACE1-INH 8, the carbonyl oxygen atom attached to the terminal $\mathrm{NH}_{2}$ group forms a $\mathrm{O} \cdot \cdots \mathrm{H}-\mathrm{O}$ H-bond with $\mathrm{Thr}_{314}$. This last interaction could not be established without the previous disruption of the $\left(\mathrm{Ala}_{313}\right) \mathrm{O} \cdot \cdot \mathrm{H}-\mathrm{O}$ $\left(\mathrm{Tyr}_{7}\right) \mathrm{H}$-bond because of the orientation of the $\mathrm{Thr}_{314}$ methyl group in BACE1-INH 8 complex, which prevents the approach of $\mathrm{Tyr}_{7}$ over the $\mathrm{Ala}_{313}$ backbone (see Figures 5(f) and 7). Moreover, in BACE1-INH 4 complex, the terminal $\mathrm{NH}_{2}$ group is forming a $\mathrm{N}-\mathrm{H} \cdots \pi$ H-bond with $\mathrm{Tyr}_{7}$ (see Figure 5(a)).

It is worth noting that unlike BACE1-INH 8 complex, in BACE1-INH 4 complex, the Ac-terminal peptide fragment is not large enough to interact directly or indirectly with F-loop residues. In BACE1--INH 8 complex, the interaction of the $\mathrm{NH}_{2}$-terminal fragment with $\mathrm{Thr}_{314}$ promotes the opening of the F-loop that helps the Ac-terminal fragment to be more deeply inserted between both loops and vice versa. In BACE1-INH 4 complex, the lack of these correlated displacements of the F-loop and the Ac-terminal fragment leads to the weaker insertion of this fragment (and also the shorter separation distance between both loops) and might explain, at least in part, the poor \% inhibition value (less than half) showed by this peptide as compared to BACE1-INH 8 complex.

Finally, INH 3 would have no effect (neither enhancing nor weakening) on the $\left(\mathrm{Ala}_{313}\right) \mathrm{O} \cdots \mathrm{H}-\mathrm{O}\left(\mathrm{Tyr}_{7}\right) \mathrm{H}$-bond because this peptide has no other residues in its $\mathrm{NH}_{2}$-terminal fragment that help to strengthen this H-bond.

\subsection{Possible role of the peptides in the BACE1 modulation}

An acceptable correlation between the sum of the charge density values at the BCPs of the network of interactions established in the BACE1-INH complex $\left(\sum \rho_{(r)}\right)$ and \% inhibition values was found.

Since the presence of the Ac-terminal fragment is critical for modulatory activity, it is reasonable to think that this peptide fragment might be involved in such conformational changes. It has been shown that the conformational change of the F-loop determines the opening or closing of the cleft between the D- and F-loops. Among both conformations, the open form seems to be the more favorable for enzyme inhibition because it allows a more efficient binding of the Ac-terminal peptide fragment within the loops. INH 8, the peptide with the highest $\%$ inhibition value, combines an open conformation of the loops with one of the largest $\sum \rho_{(r)}$ values.

\subsection{Designing new peptides with modulatory activity. Experimental corroboration}

In order to design new peptides that might modulate the exosite of BACE1, we take the peptide $\mathbf{8}$ as starting 
structure. Both the results obtained from MM GBSA as well as those obtained from the QTAIM studies were considered for the design of these new peptides. MMGBSA results (Figure 8) allowed us to determine which are the main amino acids involved in the main interactions of the different complexes. In addition, the results from QTAIM study (Figure 4) allowed us to quantify the different interactions. These results suggest that the amino acids that have the weakest interactions are Pro6, Phe8, Ile9, and Pro 10, and therefore they are the amino acid candidates to be replaced. Our simulations showed that replacements on both Pro residues introduce profound conformational changes, and therefore the presence of these two amino acids appears to be essential in order that the compounds can adopt the biologically relevant conformation. These results indicated that it would not be advisable to replace the Pro residues for the search of new INHs. Based on these results, we performed various replacements on Phe8 and Ile9. Among the simulated compounds, caught our attention mainly was the two peptides (compounds 9 (Ac-Tyr5-Pro6-Tyr7Phe8-Asp9-Pro10-Leu11- $\mathrm{NH}_{2}$ ) and 10 (Ac-Tyr5-Pro6Tyr7-Asp8-Ile9-Pro10-Leu11- $\mathrm{NH}_{2}$ )).

In the next step, we performed for peptides $\mathbf{9}$ and $\mathbf{1 0}$ all the same calculations that for the rest of the series in order to compare the results. Our theoretical calculations predict for peptides $\mathbf{9}$ and $\mathbf{1 0}$ inhibitory effects around 70 and $68 \%$, respectively. Thus, we considered interesting to synthesize and test the inhibitory activity of compounds $\mathbf{9}$ and $\mathbf{1 0}$ for two reasons. The first reason, which is obvious, is to obtain new small-size peptides possessing inhibitory activity against BACE1 acting at its exosite. The second reason is to corroborate experimentally
9 and $\mathbf{1 0}$ was performed as was described in the experimental section.

Our experimental measurements indicated that compounds 9 and $\mathbf{1 0}$ possess \% inhibition values of 20.7 and 33.1 at $10 \mu \mathrm{M}$, respectively. It should be noted that the inhibitory effects obtained for compounds $\mathbf{9}$ and $\mathbf{1 0}$ are lower than those reported for peptide $\mathbf{8}$; however, these new peptides displayed a significant modulatory activity against the exosite of BACE1. Although the experimental results are somewhat different to those predicted by the theoretical simulations, considering the different experimental conditions, such results are very encouraging. It is evident that the inhibitory effects of peptides 9 and $\mathbf{1 0}$ are less than those predicted by molecular modeling; however, at least qualitatively, these theoretical calculations indicate that peptides could have modulatory effects. This result is a support for the molecular modeling studies performed here to assist the design of new modulating agents acting on the exosite of BACE1.

Finally, a question which might arise is about the possible bioavailability of the designed small-size peptides in body/brain. In order to obtain more information on the physical-chemical characteristics of these peptides, we have calculated their $\operatorname{cog} \mathrm{P}$. Clog $\mathrm{P}$ values are $-.002,9$ for peptide 9 and -1.19 for peptide 10. Such values indicate that these peptides tend to have low lipophilicity that might hamper their absorption. While these values are acceptable for an initial structure, however, it is important to note that in terms of bioavailability, stability, and pharmacokinetics, most peptides are as bad as proteins, and, in general, they do not make good drugs unless modified in some way. It is clear that peptides possess significant limitations to be used directly as drugs; however, on the basis of our results, these peptides seems to be adequate starting structures to develop potential new modulators of BACE1

\section{Conclusions}

In this article, we report two new peptides Ac-Tyr5Pro6-Tyr7-Phe8-Asp9-Pro10-Leu11-NH2 and Ac-Tyr5Pro6-Tyr7-Asp8-Ile9-Pro10-Leu11-NH2 possessing modulatory effects on the exosite of BACE1. This activity might be considered moderate; however, it is enough significant as to test if these peptides can act in a synergistic way jointly with the well-known INHs of the catalytic site of BACE1. These studies are currently being conducted in our laboratory and will be reported soon. Besides, the fact that these compounds act on a different site of action to that of the INHs of the catalytic site is in itself promising for the development of new INHs of this enzyme.

It is interesting to note that these peptides have been obtained through a study of molecular modeling. Thus, by combining MD simulations with DFT calculations, plex BACE1-INH 8 with respect to the inhibitor amino according to the MM-GBSA method.

Notes: The $x$-axis denotes the residue number of INH and the $y$-axis denotes the interaction energy between the inhibitor and BACE1 upon binding. 
a simple and generally applicable procedure to evaluate the binding energies of small-size peptides interacting with the BACE1-exosite has been used here. This analysis provided a clear picture of the binding interactions of these peptides from a structural point of view. A correlation between binding energies obtained from DFT calcu10 lations and inhibitory effect was obtained. It must be pointed out that although the correlations obtained between the theoretical and experimental data are significant, they are not accurate enough to properly explain the different activities obtained for compounds possess-

15 ing similar inhibitory activity. Our results indicate that such differences can be better explained only from a more exhaustive electronic analysis provided by a QTAIM analysis. Thus, the results of this study provide a detailed topological description of the interaction network of the small peptides in the exosite of BACE1 and illustrate the convenience of going beyond the concept of binding energy and their relationship with the \% inhibition, in order to "see" the electronic effects within the intricate biological environment. While this kind of approaches that involve the characterization of the intermolecular interactions by means of QTAIM was traditionally applied to the study of non covalent interactions in small molecules in gas phase, we have shown here that this methodology is also a very powerful tool for 30 the study of ligand-receptor complexes, providing a very detailed description of the binding event.

\section{Abbreviations}

DCC

HOBt

35 DCM

DMF

DIEA

TFA

TIS

40 BACE1

DFT

QTAIM

Dicyclohexylcarbodiimide

Hydroxybenzotriazole

Dichloromethane

Dimethylformamide

Diisopropylethylamine

Trifluoroacetic acid

Triisopropylsilane

$\beta$-site APP Cleaving Enzyme 1

Density Functional Theory

Quantum Theory of Atoms in Molecules

\section{Supplementary material}

The supplementary material for this paper is available online at http://dx.doi.org/10.1080/07391102.2016. 1145143.

\section{Acknowledgments}

E.L.A and L.J.G. are postdoctoral fellow researcher of CONICET-Argentina. R. D. Enriz, H. A. Baldoni, and N. M. Peru- chena are members of the Consejo Nacional de Investigaciones Científicas y Técnicas (CONICET-Argentina) staff.

\section{Funding}

This work was supported by the Universidad Nacional de San Luis (UNSL); SECYT-UNNE; PIP 095 CONICET.

\section{Disclosure statement}

No potential conflict of interest was reported by the authors.

\section{References}

Andujar, S. A., Tosso, R. D., Suvire, F. D., Angelina, E., Peruchena, N., Cabedo, N., ... Enriz, R. D. (2012). Searching the "biologically relevant"conformation of dopamine: A computational approach. Journal of Chemical Information and Modeling, 52, 99-112. doi:10.1021/ci2004225

Angelina, E. L., Andujar, S. A., Tosso, R. D., Enriz, R. D., \& Peruchena, N. M. (2014). Non-covalent interactions in receptor-ligand complexes. A study based on the electron charge density. Journal of Physical Organic Chemistry, 27, 128-134. doi:10.1002/poc.3250

Atwal, J. K., Chen, Y., Chiu, C., Mortensen, D. L., Meilandt, W. J., Liu, Y., ... Watts, R. J. (2011). A therapeutic antibody targeting BACE1 inhibits amyloid- $\beta$ production in yivo. Science Translational Medicine, 3, 84ra43. doi:10. 1126/scitranslmed.3002254

Bader, R. F. W. (1985). Atoms in molecules. Accounts of Chemical Research, 18, 9-15. doi:10.1021/ar00109a003

Case, D. A., Darden, T. A., Cheatham, T. E., Simmerling, C. L., Wang, J., Duke, R. E., ... Kollman, P. A. (2012). Amber 12. San Francisco, CA: University of California.

Castillo, N., \& Boyd, R. J. (2005). A theoretical study of the fluorine valence shell in methyl fluoride. Chemical Physics Letters, 403, 47-54. doi:10.1016/j.cplett.2004.12.089

Chen, J.-Z., Yang, M.-Y., Yi, C.-H., Shi, S.-H., \& Zhang, Q.-G. (2009). Molecular dynamics simulation and free energy calculations of symmetric fluoro-substituted diol-based HIV-1 protease inhibitors. Journal of Molecular Structure: THEOCHEM, 899(1-3), 1-8. doi:10.1016/j.theochem.2008.11. 029

Clippingdale, A. B., Wade, J. D., \& Barrow, C. J. (2001). The amyloid-beta peptide and its role in Alzheimer's disease. Journal of Peptide Science, 7, 227-249. doi:10.1002/ psc. 324

Cummings, J. (2010). What can be inferred from the interruption of the semagacestat trial for treatment of Alzheimer's disease? Biological Psychiatry, 68, 876-878. doi:10.1016/ j.biopsych.2010.09.020

Essmann, U., Perera, L., Berkowitz, M. L., Darden, T., Lee, H., \& Pedersen, L. G. (1995). A smooth particle mesh Ewald method. 103, 31-34.

Frisch, M. J., Trucks, G. W., Schlegel, H. B., Scuseria, G. E., Robb, M. A., Cheeseman, J. R., ... M. C. (2009). Gaussian 09. Wallingford, CT: Gaussian.

Gutierrez, L. J., Enriz, R. D., \& Baldoni, H. A. (2010). Structural and thermodynamic characteristics of the exosite binding pocket on the human BACE1: A molecular modeling approach. The Journal of Physical Chemistry. A, 114, 10261-10269. doi:10.1021/jp104983a

Gutiérrez, L. J., Andujar, S. A., Enriz, R. D., \& Baldoni, H. A. (2013). Structural and functional insights into the antiBACE1 Fab fragment that recognizes the BACE1 exosite. Journal of Biomolecular Structure \& Dynamics. doi:10.1080/07391102.2013.821024 
Hardy, J., \& Selkoe, D. J. (2002). The amyloid hypothesis of Alzheimer's disease: Progress and problems on the road to therapeutics. Science, 297, 353-356. doi:10.1126/science. 1072994
Jabłoński, M., \& Palusiak, M. (2010). Basis set and method dependence in quantum theory of atoms in molecules calculations for covalent bonds. The Journal of Physical Chemistry. A, 114, 12498-12505. doi:10.1021/jp106740e

James Stewart. (2012). MOPAC 2012. Colorado Springs, CO. Retrieved from http://OpenMOPAC.net

Jorgensen, W. L., Chandrasekhar, J., Madura, J. D., Impey, R. W., \& Klein, M. L. (1983). Comparison of simple potential functions for simulating liquid water. The Journal of Chemical Physics, 79, 926. doi:10.1063/1.445869

Keith, T. A. (2012). AIMAll. Overland Park, KS. Retrieved from http://aim.tkgristmill.com

Kollman, P. A., Massova, I., Reyes, C., Kuhn, B., Huo, S., Chong, L., ... Cheatham, T. E. (2000). Calculating structures and free energies of complex molecules: Combining molecular mechanics and continuum models. Accounts of Chemical Research, 33, 889-897. doi:10.1021/ $\operatorname{ar} 000033 \mathrm{j}$

Kornacker, M. G., Lai, Z., Witmer, M., Ma, J., Hendrick, J., Lee, V. G., ... Copeland, R. A. (2005). An inhibitor binding pocket distinct from the catalytic active site on human $\beta$-APP cleaving enzyme. Biochemistry, 44, 11567-11573. doi:10.1021/bi0509321

Lindorff-Larsen, K., Piana, S., Palmo, K., Maragakis, P., Klepeis, J. L., Dror, R. O., \& Shaw, D. E. (2010). Improved sidechain torsion potentials for the Amber ff99SB protein force field. Proteins, 78, 1950-1958. doi:10.1002/prot.22711

Matta, C. F. (2010). How dependent are molecular and atomic properties on the electronic structure method? Comparison of Hartree-Fock, DFT, and MP2 on a biologically relevant set of molecules. Journal of Computational Chemistry, 31, 1297-1311. doi:10.1002/jcc. 21417

Párraga, J., Cabedo, N., Andujar, S., Piqueras, L., Moreno, L., Galán, A., ... Cortes, D. (2013). 2,3,9- and 2,3,11-Trisubstituted tetrahydroprotoberberines as D2 dopaminergic ligands. European Journal of Medicinal Chemistry, 68, 150-166. doi:10.1016/j.ejmech.2013.07.036

Riu, J., \& Rius, F. X. (1996). Assessing the accuracy of analytical methods using linear regression with errors in both axes. Analytical Chemistry, 68, 1851-1857. doi:10.1021/ ac951217s

Ryckaert, J., Ciccotti, G., \& Berendsen, H. J. C. (1977). Numerical integration of the Cartesian equations of motion of a system with constraints: Molecular dynamics of n-alkanes. Journal of Computational Physics, 341, 321-341.

Tosso, R. D., Andujar, S. A., Gutierrez, L., Angelina, E., Rodríguez, R., Nogueras, M., ... Enriz, R. D. (2013). Molecular modeling study of dihydrofolate reductase inhibitors. molecular dynamics simulations, quantum mechanical calculations, and experimental corroboration. Journal of Chemical Information and Modeling, 53, 2018-2032. doi:10.1021/ci400178h

Varghese, J. (2010). Wiley: BACE: Lead target for orchestrated therapy of Alzheimer's disease (p. 266). Retrieved from http://www.wiley.com/WileyCDA/WileyTitle/productCd-04 7029342X.html

William, P., Saul, T., William, V., \& Brian, F. (1986). Numerical recipes in FORTRAN 77: The art of scientific computing: fortran numerical recipes. New York, NY.

Zhou, L., Chavez-Gutierrez, L., Bockstael, K., Sannerud, R., Annaert, W., May, P. C., ... De Strooper, B. (2011). Inhibition of beta-secretase in vivo via antibody binding to unique loops (D and F) of BACE1. Journal of Biological Chemistry, 286, 8677-8687. doi:10.1074/jbc.M110.194860
35

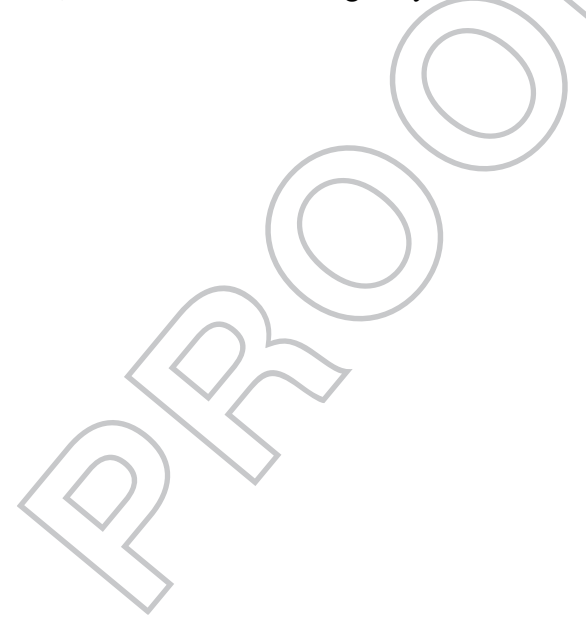

